\title{
Can a "propelling" disc stay trapped near co-rotation?'
}

\section{Caroline R. D'Angelo*}

Anton Pannekoek Institute, University of Amsterdam, Amsterdam, The Netherlands

E-mail: c.r.dangelo@uva.nl

\section{Hendrik C.Spruit}

Max Planck Institute for Astrophysics, Garching, Germany

E-mail: henk@mpa-garching.mpg.de

A strong stellar magnetic field significantly alters the behaviour of an accretion disc around the star. In particular, at low accretion rates it is often assumed that the interaction between the field and the disc will expel the gas out of the disc in an outflow rather than allow it to accrete onto the star - a state known as the "propeller regime". However, recent work has suggested that if the disc is truncated close to the co-rotation radius (where the star's spin frequency equals the Keplerian frequency of the disc) the disc-field interaction will mostly confine the gas, preventing it from escaping from the disc. The interaction changes the density profile of the disc, and can completely halt accretion onto the star without producing an outflow. In turn, this forces the inner edge of the disc to stay trapped close to the co-rotation radius even when there is no net mass flow through the disc, so that the disc never moves into the true propeller regime. Below I explain how a trapped disc can form and show how this can sometimes result in bursts of accretion onto the star, such as have been observed as low-frequency QPOs in X-ray pulsars SAX J1808.4-3658 and NGC 6440 X-2. Finally, I discuss how trapped discs could also be responsible for the weak recurrent outbursts seen in NGC 6440 X-2 and IGR J00291+5934.

Fast X-ray timing and spectroscopy at extreme count rates: Science with the HTRS on the International $X$-ray Observatory - HTRS2011,

February 7-11, 2011

Champéry, Switzerland

\footnotetext{
* Speaker.
} 


\section{Introduction}

The strong stellar magnetic fields of neutron stars exert a large influence on gas surrounding them, affecting both observational properties and evolution of the accreting system. In the inner regions of the accretion flow the magnetic field regulates the flow and funnel accretion along field lines onto the surface of the star, which is observed as pulsed X-ray emission as a result of the rotating hot spot(1). Some X-ray pulsars show an evolution in their period, spinning up or down, an evolution usually attributed to interactions between the magnetic field and the surrounding accretion flow (e.g. (2)).

Many details surrounding the interaction between matter in a Keplerian accretion disc and a strong stellar magnetic field remain uncertain, and a number of accreting neutron stars show unexplained behaviour that could be a result of this interaction. For example, the outbursting Xray pulsar, SAX J1808.8-3658, has shown a high-amplitude, $1 \mathrm{~Hz}$ quasi-periodic oscillation in the decay phase of several outbursts (3), a behaviour that has also recently been observed in another X-ray pulsar, NGC 6440 X-2, (4). The comparatively low frequency of the QPO suggests that it might be regulated by viscous timescales in the inner accretion disc as opposed to e.g. the rotation frequency of the star, and hence caused by interaction between the magnetic field and the disc. NGC 6440 X-2 also undergoes weak, recurrent outbursts (5) on much shorter timescales (around 30 days) than predicted by a standard outburst model. This suggests that a significant amount of mass remains in the disc during quiescence in order to fuel the outburst. As discussed below, both these observations can be explained by a revised understanding of how an accretion disc interacts with a magnetic field, especially at low accretion rates.

\subsection{Magnetically-regulated accretion}

A strong stellar magnetic field regulates the trajectory of infalling ionized matter in the region close to the star. The distance at which gas is controlled by the field is usually termed the magnetospheric radius, $r_{\mathrm{m}}$. For spherically-symmetric infall, this radius can be estimated by equating the ram pressure of the infalling gas to the magnetic pressure (6):

$$
r_{\mathrm{m}} \sim\left(\frac{B_{\mathrm{S}}^{2} R_{*}^{6}}{\left(G M_{*}\right)^{1 / 2} \dot{M}}\right)^{2 / 7},
$$

where $B_{\mathrm{S}}$ is the star's surface dipole field, $R_{*}$ and $M_{*}$ are respectively the star's radius and mass, and $\dot{M}$ is the accretion rate.

In stars accreting from a Keplerian disc, this radius must be modified to account for the disc's angular momentum. The modified radius can be estimated by considering the azimuthal equation of motion for the gas in the disc at the point where it is forced to co-rotate with the star (7)):

$$
r_{\text {in }}=\left(\frac{\eta B_{\mathrm{S}}^{2} R_{*}^{6}}{4 \Omega_{*} \dot{M}_{\text {in }}}\right)^{1 / 5} .
$$

In this equation, $\Omega_{*}$ is the spin frequency of the star, and $\eta \equiv B_{\phi} / B_{\mathrm{Z}}$, which is a measure of the azimuthal magnetic field component $\left(B_{\phi}\right)$ induced by differential rotation between the star and the disc in the field lines that connect the two. This estimate of $r_{\text {in }}$ is typically somewhat smaller than that given by eq. 1.1. 
The relative rotation between the star and the disc causes angular momentum exchange along the coupled field lines. The amount of angular momentum exchange depends, among other things, on the extent of the disc that is coupled to the star. Both analytic arguments (e.g. (8;9)) and numerical simulations (such as (10)) suggest that the coupled field lines are rapidly wound up by the differential rotation between star and disc, causing them to inflate and open. This limits the growth of the induced $B_{\phi}$ component of the field to $B_{\phi}=\eta B_{z}$, with $\eta$ of order unity, and reduces the radial extent of the interaction region, since beyond a given radius the field lines are always open and the disc-field connection will be severed. We assume the interaction region to be small, with $\Delta r / r<1$.

Whether the star loses or gains angular momentum will depend on the co-rotation radius, $r_{\mathrm{c}}$, the radius at which the spin frequency of the star equals the Keplerian frequency of the disc:

$$
r_{\mathrm{c}} \equiv\left(\frac{G M_{*}}{\Omega_{*}^{2}}\right)^{1 / 3}
$$

If $r_{\text {in }}<r_{\mathrm{c}}$, angular momentum is removed from the disc and accretion can proceed. However, if $r_{\mathrm{c}}>r_{\text {in }}$ angular momentum is added to the disc, creating a centrifugal barrier that inhibits accretion. This is usually termed the "propeller" regime, under the assumption that the material in the disc will be expelled from the system when it reaches $r_{\text {in }}$ (11). As we argue below, this description of the disc when $r_{\mathrm{c}}>r_{\text {in }}$ is simplistic and likely sometimes at least incorrect.

\section{Magnetic accretion at low $\dot{M}$ : "propellers", outflows and dead discs}

Above we presented a standard argument used to estimate the inner radius of an accretion disc in the presence of a magnetic field (eq. 1.2). This calculation presupposes that there is accretion through the inner edge of the disc. However, when $r_{\text {in }}>r_{\mathrm{c}}$, the interaction between the disc and the field creates a centrifugal barrier that opposes accretion onto the star, so this reasoning is invalid. Matter will continue to move inwards from large radii, but will be prevented from accreting onto the star. What then happens in this case?

A common assumption is that once $r_{\text {in }}>r_{\mathrm{c}}$, the interaction between the disc and the magnetic field will completely expel the mass at $r_{\text {in }}$ in an outflow. This is known as the propeller regime, with the spinning magnetic field likened to a propeller flinging matter out of the system (11). The propellered disc 'solves' the problem presented by 1.2 by assuming that when $r_{\text {in }}>r_{\mathrm{c}}$ the matter moving through the disc will be expelled rather than accreted.

It is not clear what the accretion rate (if any) onto the star should be in the propeller regime, although one would assume that it should decrease sharply around $r_{\mathrm{in}}=r_{\mathrm{c}}$ if most of the mass flowing through the disc is being expelled in an outflow. Despite this, observational results often refer to sources as being in the propeller regime, meaning only that $r_{\mathrm{c}} 1.3$ is larger than $r_{\text {in }}$ (as calculated using 1.1 or similar for the observed accretion rate). This clearly does not make sense: if the ratio of accreted to outflowing matter were very small, then the total accretion rate through the disc (incorporating the unobserved outflow) could be large enough to make 1.1 smaller than 1.3 !

The problem is caused by what is understood by propeller regime. The original suggestion referred to systems in which the disc is truncated at a considerable distance outside $r_{\mathrm{c}}$, so that 
$\Omega_{*} \gg \Omega_{\mathrm{K}}\left(r_{\text {in }}\right)$. In this case the rotational energy of the magnetosphere is much larger than that of the disc, so the majority of the disc mass is likely expelled by the disc-field interaction. However, in order to avoid a logical problem (i.e. what happens if most but not all the mass is expelled at $r_{\text {in }}$ ?), models of the propeller regime are typically constructed to ensure that the disc is completely expelled in a magnetic outflow by the time it reaches $r_{\text {in }}$. This is in spite of the fact that the physics of powering magnetic outflows (e.g. how much energy is required, or how much mass can be expelled) is still strongly uncertain.

Further adding to the confusion is the tendency (as noted above), to define the propeller regime as $r_{\text {in }}>r_{\mathrm{c}}$ as determined by 1.1 and 1.3, despite the fact that the definition of a propeller implies a system in which matter is expelled rather than accreted. The logic is thus: a) the centrifugal barrier at $r_{\text {in }}$ will prevent accretion onto the star, $\mathrm{b}$ ) there is (probably) enough energy in the rotation of the magnetosphere to expel the disc in an outflow, c) since the accretion rate in the disc is determined far from $r_{\text {in }}$, the disc must be expelled, otherwise, where would the mass go?

This logic fails most obviously for cases where $r_{\mathrm{c}}<r_{\text {in }}<1.26 r_{\mathrm{c}}$, where the rotation rate of the magnetosphere is less than the escape speed of the gas. As the gas at $r_{\text {in }}$ is brought into corotation with the star, the added rotational energy will not be enough to expel it, but the centrifugal barrier will prevent it from accreting onto the star (7). The same condition could of course hold for a disc truncated even further from $r_{\mathrm{c}}$ : having enough energy for the gas to escape is not sufficient to require that the disc be expelled, and some of the gas at $r_{\text {in }}$ could remain confined in the disc.

The solution to this problem is that the standard disc solution that describes accretion onto a star or magnetosphere (12) does not necessarily apply to discs truncated outside $r_{\mathrm{c}}$. This is not such a problem as it initially appears, since there are in fact a whole class of time-independent accretion disc solutions with different boundary conditions at $r_{\text {in }}$. The most well-known of these is the solution for a disc accreting onto the surface of a non-magnetic star spinning close to break-up (13), where the disc surface density structure changes to carry away the excess angular momentum from the star while accretion continues.

A similar disc solution called a "dead disc" exists for the case where accretion onto the star is completely suppressed but the disc-field interaction adds angular momentum to the inner edge of the disc $(14 ; 15))$. The inner edge of a dead disc is determined by the location where the rate of angular momentum being injected into the disc at $r_{\text {in }}$ balances the rate at which the disc can transport it outwards. In a dead disc, the inner radius is thus determined by the amount of mass in the disc, rather than the accretion rate through it. More mass in the inner parts of the disc means that the disc can transport more angular momentum outwards, and $r_{\text {in }}$ will move to $r_{\mathrm{c}}$.

If the average accretion rate falls enough that accretion onto the star is suppressed, then mass can begin to pile up in the inner regions of the disc. This will last until there is enough mass in the disc to push $r_{\text {in }}$ inside $r_{\mathrm{c}}$, where the accumulated mass can be accreted. The result will be bursts of accretion onto the star, with the timescale regulated by the viscous diffusion timescale in the inner regions of the disc $(15 ; 7)$.

A dead disc solution will manifest in other ways in addition to accretion bursts. For example, the disc will remain truncated very close to $r_{\mathrm{c}}$ as long as there is any accretion onto the star, which allows the star to be efficiently spun down by the disc.

The following sections briefly present our investigations of these accretion bursts and the longterm evolution of a dead disc. This work is presented in greater detail in $(14 ; 16 ; 17)$. 

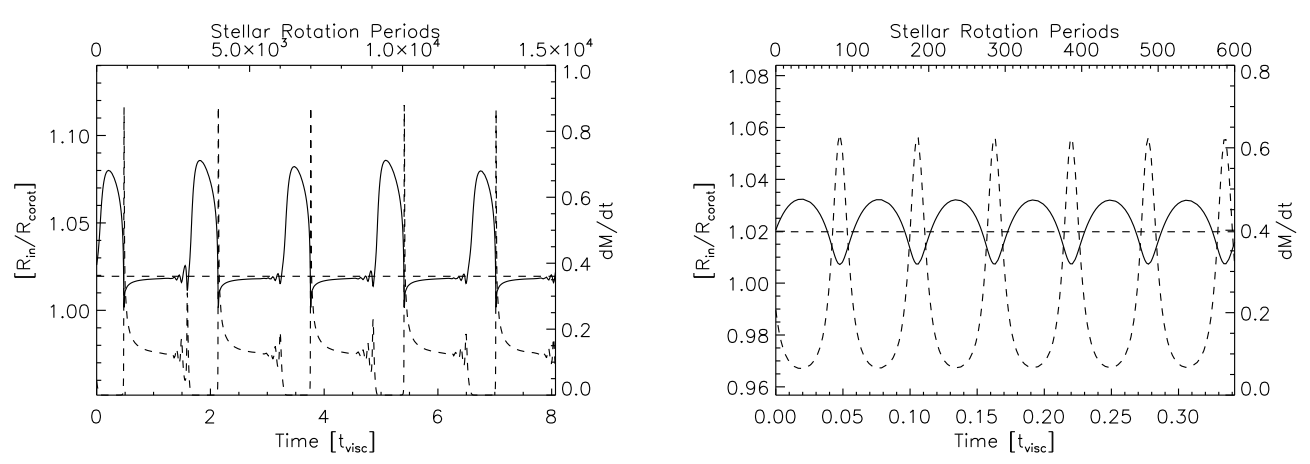

Figure 1: Accretion instability observed in our simulations. The solid line shows the change in disc inner radius, while the dashed line shows the change in accretion rate onto the star with respect to $\dot{m}_{\mathrm{c}}$, the accretion rate that would put $r_{\text {in }}$ at $r_{\mathrm{c}}$.. LEFT: The low-frequency region of instability. The disc moves through distinct periods of outburst and quiescence and the outburst profile takes the form of a relaxation oscillator. RIGHT: The high-frequency region of instability. In addition to a much higher frequency of oscillation, the instability has a smoother outburst profile, and accretion continues even during the low phase of the instability.

\section{Episodic Accretion Bursts}

As the accretion rate decreases, the inner edge of the disc moves outward until the centrifugal barrier increases enough to suppress accretion onto the star. This can lead to an instability at the inner edge of the disc, where material periodically piles up and is dumped onto the star. This instability was investigated in $(7 ; 14 ; 17)$.

The properties of the instability are determined by the mean accretion rate onto the star, the width of the coupling between the star and disc, and the radial extent around co-rotation around which the disc moves from accreting to non-accreting. These three variables determine whether the instability appears (14), its period, and the shape and duty cycle of the accretion burst. Figure 1 shows some sample bursts.

In DS11b we found two distinct regions of instability, shown in figure 1. In the first region (left panel), accretion proceeds as outlined above, and accretion onto the star is mostly suppressed while matter builds up. The period of the outburst increases with decreasing mean accretion rate, since a lower accretion rate requires a longer time to build up enough material in the disc to power an outburst. The period of the outbursts can vary over four orders of magnitude, from $\sim 0.1-$ $100 T_{\text {visc }}$, where $T_{\text {visc }}$ is the viscous timescale at $r_{\mathrm{c}}$. The outburst shape also changes substantially with changing accretion rate, although it is most sensitive to the conditions on the inner boundary (our other two parameters). The outburst profile is typically a relaxation oscillator, with an initial spike followed by a longer tail, although higher-frequency oscillations within the outburst are also frequently observed.

The second region of instability (right panel) occurs when the mean accretion rate is high enough to keep $r_{\text {in }}$ close to $r_{\mathrm{c}}$. The instability has a much higher frequency than the first region (with periods between $\sim 0.01-1 T_{\text {visc }}$, and the disc continues to accrete (although at a much lower rate) throughout the instability. The shape of the outburst also changes, with a smooth transition between high and low accreting states. It seems likely that the high-frequency oscillations observed 

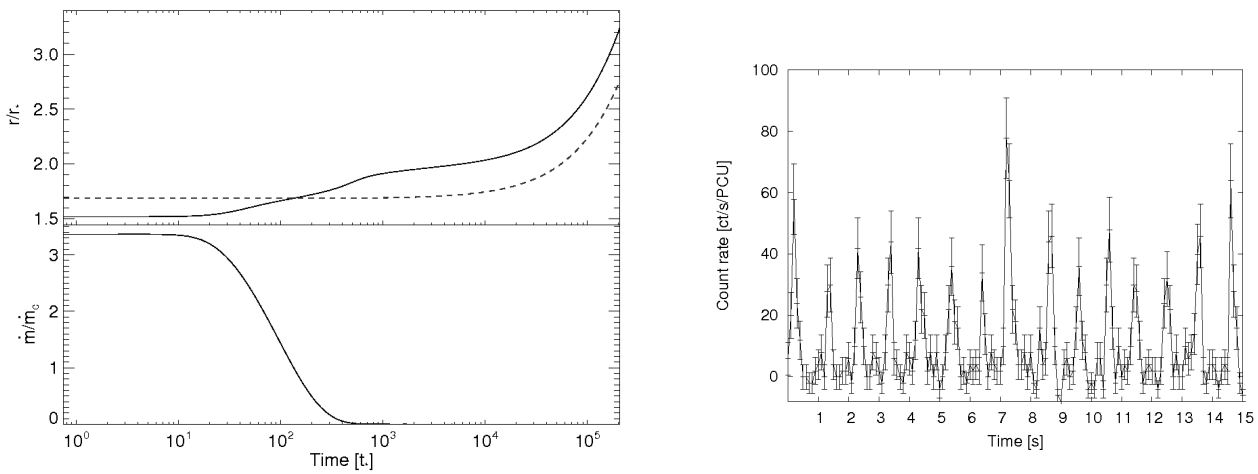

Figure 2: LEFT: The transition from an accreting to a trapped disc. The top panel shows the inner radius (solid line) and co-rotation radius (dashed line) evolution as a function of time. As the star spins down, the co-rotation radius moves outward, and is tracked by the inner radius of the disc. The bottom panel shows the decreasing accretion rate onto the star as the disc enters the trapped regime. RIGHT: The 1-Hz QPO detected from SAX J1808.4-3658. The strongly pulsed signal could be caused by the disc instability presented in this paper. Figure from (3).

in some outbursts in the first instability region are this higher frequency instability. Since this instability occurs exactly around the transition between accreting and non-accreting states, the detailed properties of the instability are likely sensitive to the way in which the transition takes place, which in our simulation is parameterized by two numerical parameters.

\section{Trapped Discs}

The accretion instability will appear when there is a mismatch between the accretion rate at $r_{\text {in }}$ and that further out in the disc. However, we can also study what will happen when the accretion rate in the disc falls close to zero. In the traditional accretion picture zero accretion rate means that there is no accretion in the disc, but in the picture outlined here, mass can be retained in the disc even when no gas is accreted or expelled from the system.

In DS11a we studied the evolution of the disc-star system under these conditions. Since the interaction between the disc and the star removes angular momentum from the star, the long-term evolution of the system will cause the star to slow down and the co-rotation radius to move outward. In many cases, we found that the inner edge tracks the co-rotation radius and the two move outward together, with a nearly constant ratio $r_{\text {in }} / r_{\mathrm{c}}$. We refer to this as a "trapped disc", since the disc inner radius remains trapped close to $r_{\mathrm{c}}$ as the star spins down. This behaviour is shown in the left panel of figure 2 .

The long-term evolution of the star's spin rate will depend on the conditions in the disc, which must be large enough to absorb the star's angular momentum, or coupled to a sink of angular momentum at its outer edge (such as a binary companion). The ratio of characteristic spin-down and viscous timescales in the system will also affect its evolution. The spin-down timescale can be estimated by calcuating the changing torque on the star as it spins down. For a dipolar magnetic 
field, the timescale to spin down to $\Omega_{f}$ is:

$$
T_{\mathrm{SD}} \sim \frac{G M_{*} I}{\Omega_{f} \mu^{2}}
$$

If this timescale is too long compared with the viscous timescale, $T_{\mathrm{visc}}$, the disc will move outward faster than $r_{\mathrm{c}}$ and the spin down of the star will cease.

\section{Application to Accreting Neutron Stars}

Several magnetically accreting sources show behaviour that could be accounted for by the formation of a trapped disc and subsequent periodic accretion bursts.

At least two accreting X-ray pulsars, NGC 6440 X-2 (18) and IGR J00291+5934 (19) have been observed to undergo weak, recurrent outbursts on much shorter timescales (around 30 days) than would be predicted from an ionization-instability model (20). This suggests that a significant amount of mass is retained in the inner disc during quiescence, i.e. that the disc becomes trapped.

IGR J00291+5934 is an X-ray pulsar that historically has shown standard single outbursts (in 1998, 2001, and 2004) followed by long periods of quiescence. However, in 2008 it underwent a substantially different outburst, with two weaker outbursts (respectively lasting nine and 13 days) separated by 27 days of quiescence (19). The authors estimated that the total mass flux of weaker outbursts together was approximately equal to the single 2004 outburst. Similar outbursts have been seen in NGC $6440 \mathrm{X}-2$, a recently discovered X-ray binary with a frequency of $442 \mathrm{~Hz}$ in a 57 minute orbit (5). Since discovery on July 28, 2009, five distinct outbursts have been observed, each typically lasting around four days at a luminosity of around $L_{X} \sim 10^{35} \mathrm{erg} \mathrm{s}^{-1}$, with a minimum recurrence time of around 31 days.

The short recurrence timescales suggest that a considerable amount of mass is stored in the disc when the accretion rate drops, which can then fuel another outburst when $\dot{m}$ increases again. In both sources the quiescent X-ray luminosity is at least 4 orders of magnitude lower than the outburst luminosity, indicating a large change in accretion rate (although the X-ray luminosity may not be a good indicator of bolometric luminosity at such low accretion rates, so there is some uncertainty in the change in $\dot{m}$ ).

During two outbursts, the power spectrum of NGC 6440 X-2 was dominated by a strong lowfrequency QPO $(\sim 1 \mathrm{~Hz})(4 ; 19)$. A similar QPO was previously detected in the tail an outburst in X-ray pulsar SAX J1808.4-3658 (3) which was attributed to the disc instability presented in DS10a and (7). A much weaker QPO at $0.5 \mathrm{~Hz}$ was observed in the outbursts IGR J00291+5934 and might also be attributed to the disc instability (19).

\section{Conclusion}

The interaction between a strongly magnetic star and a surrounding accretion flow substantially alters the behaviour of the accretion, especially at low accretion rates. When the accretion rate drops below a critical threshold, the interaction could halt accretion onto the star altogether, without powering a substantial outflow. This leads to the formation of a "trapped disc" in which there is little accretion but substantial mass in the circumstellar disc and the inner radius of the disc 
remains close to co-rotation. A trapped disc can efficiently spin down the star, and produce regular bursts of accretion.

This model is promising to explain the observed strong 1-Hz QPOs observed in SAX J1808.83658 and NGC 6440 X-2. It could also account for the weak and rapidly recurring outbursts seen in NGC 6440 X-2 and IGR J00291+593.

\section{References}

[1] K. Davidson and J. P. Ostriker, Neutron-Star Accretion in a Stellar Wind: Model for a Pulsed X-Ray Source, ApJ 179 (Jan., 1973) 585-598.

[2] L. Bildsten, D. Chakrabarty, J. Chiu, M. H. Finger, D. T. Koh, R. W. Nelson, T. A. Prince, B. C. Rubin, D. M. Scott, M. Stollberg, B. A. Vaughan, C. A. Wilson, and R. B. Wilson, Observations of Accreting Pulsars, ApJS 113 (Dec., 1997) 367-+, [arXiv:astro-ph/9707125].

[3] A. Patruno, A. Watts, M. Klein Wolt, R. Wijnands, and M. van der Klis, $1 \mathrm{~Hz}$ Flaring in SAX J1808.4-3658: Flow Instabilities near the Propeller Stage, ApJ 707 (Dec., 2009) 1296-1309, [0 904 .0560].

[4] A. Patruno, Y. Yang, D. Altamirano, M. Armas-Padilla, Y. Cavecchi, N. Degenaar, M. Kalamkar, R. Kaur, M. V. D. Klis, A. Watts, R. Wijnands, M. Linares, P. Casella, N. Rea, P. Soleri, C. Markwardt, T. Strohmayer, and C. Heinke, New outburst of the accreting millisecond X-ray pulsar NGC $6440 \mathrm{X}-2$ and discovery of a strong $1 \mathrm{~Hz}$ modulation in the light-curve, The Astronomer's Telegram 2672 (June, 2010) 1-+.

[5] D. Altamirano, A. Patruno, C. O. Heinke, C. Markwardt, T. E. Strohmayer, M. Linares, R. Wijnands, M. van der Klis, and J. H. Swank, Discovery of a $205.89 \mathrm{~Hz}$ Accreting Millisecond X-ray Pulsar in the Globular Cluster NGC 6440, ApJ 712 (Mar., 2010) L58-L62, [0 911.0435$].$

[6] J. E. Pringle and M. J. Rees, Accretion Disc Models for Compact X-Ray Sources, A\&A 21 (Oct., 1972) 1-+.

[7] H. C. Spruit and R. E. Taam, An instability associated with a magnetosphere-disk interaction, ApJ 402 (Jan., 1993) 593-604.

[8] J. J. Aly, Quasi-static evolution of sheared force-free fields and the solar flare problem, A\&A 143 (Feb., 1985) 19-22.

[9] R. V. E. Lovelace, M. M. Romanova, and G. S. Bisnovatyi-Kogan, Spin-up/spin-down of magnetized stars with accretion discs and outflows, MNRAS 275 (July, 1995) 244-254, [arXiv:astro-ph/9412030].

[10] A. P. Goodson, R. M. Winglee, and K. Boehm, Time-dependent Accretion by Magnetic Young Stellar Objects as a Launching Mechanism for Stellar Jets, ApJ 489 (Nov., 1997) $199-+$. 
[11] A. F. Illarionov and R. A. Sunyaev, Why the Number of Galactic X-ray Stars Is so Small?, $A \& A 39$ (Feb., 1975) 185-+.

[12] N. I. Shakura and R. A. Sunyaev, Black holes in binary systems. Observational appearance., A\&A 24 (1973) 337-355.

[13] B. Paczynski, A polytropic model of an accretion disk, a boundary layer, and a star, ApJ 370 (Apr., 1991) 597-603.

[14] C. R. D'Angelo and H. C. Spruit, Episodic accretion on to strongly magnetic stars, MNRAS 406 (Aug., 2010) 1208-1219, [1001.1742].

[15] R. A. Sunyaev and N. I. Shakura, Disk reservoirs in binary systems and prospects for observing them, Pis ma Astronomicheskii Zhurnal 3 (June, 1977) 262-266.

[16] C. D'Angelo and H. Spruit, Long-Term evolution of Discs around Magnetic Stars, ArXiv e-prints (Feb., 2011) [1102.3697].

[17] C. R. D'Angelo and H. C. Spruit, Accretion Discs Trapped Near Corotation, in prep (2011).

[18] C. O. Heinke, D. Altamirano, H. N. Cohn, P. M. Lugger, S. A. Budac, M. Servillat, M. Linares, T. E. Strohmayer, C. B. Markwardt, R. Wijnands, J. H. Swank, C. Knigge, C. Bailyn, and J. E. Grindlay, Discovery of a Second Transient Low-Mass X-ray Binary in the Globular Cluster Ngc 6440, ApJ 714 (May, 2010) 894-903, [0 911.0444 ].

[19] J. M. Hartman, D. K. Galloway, and D. Chakrabarty, A Double Outburst from IGR J00291 +5934: Implications for Accretion Disk Instability Theory, ArXiv e-prints (June, 2010) [1006.1908].

[20] J. Lasota, The disc instability model of dwarf novae and low-mass $X$-ray binary transients, New Astron. Rev. 45 (June, 2001) 449-508, [arXiv : astro-ph/0102072]. 